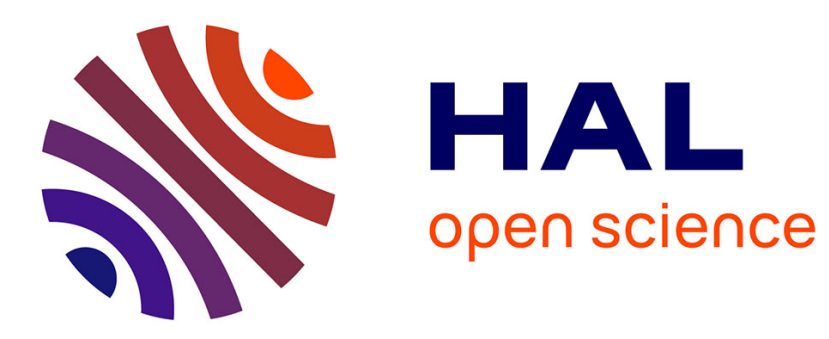

\title{
Speech production after glossectomy: methodological aspects
}

\author{
Audrey Acher, Pascal Perrier, Christophe Savariaux, Cécile Fougeron
}

\section{To cite this version:}

Audrey Acher, Pascal Perrier, Christophe Savariaux, Cécile Fougeron. Speech production after glossectomy: methodological aspects. Clinical Linguistics \& Phonetics, 2014, 28 (4), pp.241-56. 10.3109/02699206.2013.802015 . hal-00983108

\section{HAL Id: hal-00983108 \\ https://hal.science/hal-00983108}

Submitted on 24 Apr 2014

HAL is a multi-disciplinary open access archive for the deposit and dissemination of scientific research documents, whether they are published or not. The documents may come from teaching and research institutions in France or abroad, or from public or private research centers.
L'archive ouverte pluridisciplinaire HAL, est destinée au dépôt et à la diffusion de documents scientifiques de niveau recherche, publiés ou non, émanant des établissements d'enseignement et de recherche français ou étrangers, des laboratoires publics ou privés. 


\title{
Speech production after glossectomy: methodological aspects
}

\author{
Audrey Acher ${ }^{1}$, Pascal Perrier ${ }^{1}$, Christophe Savariaux ${ }^{1}$, \& Cecile Fougeron ${ }^{2}$ \\ ${ }^{1}$ GIPSA-Lab, UMR 5216 CNRS/ Université Grenoble-Alpes, \\ Saint Martin d'Hères, France \\ ${ }^{2}$ Laboratoire de Phonétique et Phonologie, UMR 7018 CNRS/Univ. Paris, France
}

Clin Linguist Phon. 2014 Apr;28(4):241-56. doi: 10.3109/02699206.2013.802015. Epub 2013 Jul 9.

\section{Abstract}

This paper focuses on methodological issues related to quantitative assessments of speech quality after glossectomy. Acoustic and articulatory data were collected for 8 consonants from two patients. The acoustic analysis is based on spectral moments and the Klatt VOT. Lingual movements are recorded with ultrasound without calibration. The variations of acoustic and articulatory parameters across pre- and post-surgery conditions are analysed in the light of perceptual evaluations of the stimuli. A parameter is considered to be relevant if its variation is congruent with perceptual ratings. The most relevant acoustic parameters are the skewness and the Center of Gravity. The Klatt VOT explains differences that could not be explained by spectral parameters. The SNTS ultrasound parameter provides information to describe impairments not accounted for by acoustical parameters. These results suggest that the combination of articulatory, perceptual and acoustic data provides comprehensive complementary information for a quantitative assessment of speech after glossectomy.

Keywords: hemiglossectomy, tongue cancer, ultrasound, acoustic analysis. 


\section{Introduction}

Malignant intraoral tumours have a high lethality and a noticeable sociopsychological impact. The plexiform musculature of the tongue facilitates the development of invasive tumours (Meley \& Barthelmé, 1987) and 6500 new cases were diagnosed in France in 2010 (Ligier et al., 2011). Clinical treatments have recently improved and a variety of possibilities are now available for the patients: chemotherapy, radiation therapy and surgery.

Surgical treatments of tongue cancers consist of a resection of the tumour and surrounding tissues, which is known as glossectomy. Depending on the size of the tumour, the glossectomy can be further classified as partial or total, and it concerns different types of adjacent structures in the tongue. Usually, if the volume of the resected tissues is large, the resection is followed by a reconstruction. During the reconstruction, a flap is grafted to the remaining tongue tissues, in order to give to the tongue a volume and a form that are similar to the one of the patient before surgery. The impact of the flap on tongue mobility depends on the volume of the resected tissues (Heller et al., 1991; Dios et al., 1994), the location of the tumour (KorpijaakkoHuukha et al., 1998), the mechanical characteristics of the flap (Buchaillard et al., 2007) and its connections to the tongue tissues (Konstantinovic \& Dimic, 1998; Bressmann, 2004, 2007).

Radiation therapy and chemotheraphy can generate fibrosis, i.e. an excess of fibrous connective tissues. This tends to increase the rigidity of the tongue and to decrease its mobility and flexibility (Bundgaard et al., 1993). It can also cause xerostomia (drying of the mucosa and the mouth). Xerostomia limits mobility, especially in cases involving contact of the tongue with the teeth or the palate. For all 
treatments, specific factors such as age and postoperative complications (Kazi et al., 2007; Laaksonen et al., 2011) can slow down and limit the speech recovery.

In summary many factors can influence the quality of speech after clinical treatments of tongue cancer. For speech therapists, quantitative methods are needed to assess speech quality in post-surgery conditions in order to develop (and later evaluate) their rehabilitation programme. Crucially, the consequences of articulatory impairments following the surgery on speech intelligibility need to be further explored in order to be improved.

The goal of this paper is to contribute to the development of a methodology for assessing speech quality, which is based on measurements of specific physical variables, in the acoustic and the articulatory domains. Findings are based on the combination of data recorded from two patients who have undergone a hemiglossectomy: the acoustic signal, ultrasound data on tongue position without calibration and perceptual assessments of speech quality.

The absence of calibration for ultrasound data is an important drawback of ultrasound studies with patients after vocal tract surgery. Their general health state does not allow the use of a helmet (such as the one manufactured by Articulate Instruments Ltd 2008) or any other device for tightly maintaining the head and the probe in a fixed position (Stone et al,. 1995). The presence of oedema in the first post-surgery condition prevents from having the same position of the probe across assessment conditions due to the temporary swelling of the neck. Solutions based on optical measures have also been proposed (Whalen et al., 2005). However, this experimental set-up was not available for this study. Consequently, part of the specificity of this work was to address the issue of extracting reliable information from non-calibrated ultrasound data. 


\section{Materials and methods}

\section{Patients}

Two native speakers of French participated in this study $(\mathrm{P} 1$, a male, 28 years old and P2, a female, 62 years old). They have undergone a partial glossectomy and a bilateral neck dissection. The right side of the tongue was resected and reconstructed using a musculocutaneous infrahyoid flap.

The resection resulted in a hemiglossectomy. A hemiglossectomy is the resection of half of the tongue longitudinally. In both patients the tip of the tongue was preserved. Patients were recorded once in pre-surgery condition (condition PREOP) and twice in post-surgery condition, namely 1 month (condition POSTOP1) and 3 months (condition POSTOP2) after surgery. Before the first pre-surgery recording (condition PREOP), both patients received chemotherapy. They started to receive radiation therapy 6 weeks after surgery (duration 6 weeks). The radiation therapy occurred between the POSTOP1 and POSTOP2 conditions. Tumours were classified as T4 according to the TNM classification of malignant tumours.

\section{Experimental Data}

The corpus was made of 24 CVCVC sequences where $C$ is one of the $/ t, d, k$, $\mathrm{g}, \mathrm{s}, \mathrm{z}, \int, 3 /$ consonants and $\mathrm{V}$ is either /i/, /u/ or /a/. Each sequence was repeated 6 times in isolation in a specified order in a quiet room in the hospital. The acoustic signal was recorded together with tongue movements measured with ultrasound. The sampling frequency for the acoustic signal was $22050 \mathrm{~Hz}$. The ultrasound machine was a Mindray DP-6600, the convex probe was a Mindray 35C20EA with a frequency 
of $3.5 \mathrm{MHz}$. The ultrasound stream rate was 60 frames per second. The experiment was run using Articulate Assistant Advanced software (AAA) version 2.09 from Articulate Instrument (Articulate Instruments Ltd 2011). The probe was placed under the chin. Lingual imaging data were collected in the coronal and the sagittal plane (three repetitions each) with the AAA software. In this study, only sagittal data were analysed.

Recently ultrasound has become more popular in phonetic research. It is noninvasive technique that does not involve any risk for the patient. In addition, portable ultrasound units have been designed at reasonable cost. It is a useful tool to describe static and dynamic aspects of the shape of the tongue during speech and swallowing (Stone 2005). The real time display of the shape of the tongue on a screen makes this technique very attractive for speech therapists as a tool for visual feedback. This technique has been used in particular to study pathological speech. Some researchers used it to investigate speech production after glossectomy (Schliephake et al., 1998, Bressmann et al., 2007).

\section{Data analysis}

The analysis included (1) extracting the spectral and temporal parameters from the acoustic signal, (2) extracting information about lingual movement and tongue positioning in the oral cavity from the ultrasound data and (3) assessing perceptually the quality of the speech produced. The focus was the analysis of the consonants /t, d, k, g, s, z, S, z/ in the centre of the CVCVC sequences, surrounded by a symmetrical vowel context $(/ \mathrm{i}-\mathrm{i} /, / \mathrm{u}-\mathrm{u} /$ and $/ \mathrm{a}-\mathrm{a} /)$. Consonantal articulations were chosen in this study because of the precise accuracy in tongue positioning and in the 
timing of the tongue movements required for their production. Hence, they are very appropriate to the purpose of this study.

\section{Perceptual evaluation}

Five speech therapists have participated in a perceptual evaluation of the speech production of the two patients. Two repetitions of each VCV sequence were presented once in a random order $(\mathrm{N}=144 \mathrm{VCV}$ per patient i.e. 8 consonants $\times 3$ vowel contexts $\times 2$ repetitions $\times 3$ conditions). The judges were asked to rank the quality of the stimuli from 1 (normal), 2 (sightly impaired), 3 (moderately impaired), 4 (strongly impaired) to 5 (unintelligible). The identity of the consonant was given.

\section{Acoustic parameters}

The acoustical characterization of all consonants was based on the computation of the spectral moments $\left(1^{\text {st }}\right.$ moment: Centre Of Gravity $(\mathrm{COG}), 3^{\text {rd }}$ moment: skewness and $4^{\text {th }}$ moment: kurtosis) (Forrest et al., 1988). Spectral moments inform about the distribution of the energy in the frequency domain. For consonants' classification, the relevance of methods based on the characterization of this energy distribution for the burst or for the frication noise is a controversial issue in the literature (see among others Forrest et al., 1988; Jesus \& Shadle, 2002; Jones \& Munhall, 2003). However, based on a number of different trials on our own data, and in line with Forrest and colleagues (1988), we decided to use and evaluate these spectral moments in the current study.

The timing of the consonantal gesture was also indirectly captured in the acoustical domain via the Klatt Voice Onset Time (VOT) (Stevens \& Klatt, 1974). As compared to the initial definition of VOT (Lisker \& Abramson, 1964), the Klatt VOT 
includes the transition from the consonant to the vowel. It is more relevant for the purpose of this study, because it accounts for the time properties of tongue movement after the consonantal release. This definition, originally proposed for stops, was extended to sibilants (see below).

The acoustical analysis requires a careful manual labelling of the signal in order to specify the segments of the signal that are relevant for the computation of the spectral moments. The labelling was achieved using simultaneously the time and the wide-band spectrographic representation. It includes four time events as shown in figure 1:

- The offset of the vowel preceding the consonant (V1); it was defined as the first zero crossing with rising edge after F2 vanishes on the spectrogram.

- The onset and offset of the noisy part of the consonant, i.e. the burst for the stops, and the frication noise for sibilants: they were determined on the spectrogram as the beginning and end of the broad band high frequency region $(4000-8000 \mathrm{~Hz})$.

- The onset of laminar flow in the vocal tract after the consonant: it is defined as the time where formant F2 re-appears clearly on the spectrogram after the onset of the noisy part of the consonant.

On the basis of these four labels, three durations were determined as seen in figure 1: (1) the total hold duration of the consonant, from V1 offset to the onset of the noisy part for the stops, and from $\mathrm{V} 1$ offset to the offset of the noisy part for the sibilant; (2) the noise duration from the onset to the offset of the noisy part of the consonant; (3) the Klatt VOT from the end of the consonant hold part (i.e. the onset of the noisy part for the stops and the offset of the noisy part for the sibilants) to the 
onset of the laminar flow. In this study, only the noise duration and Klatt VOT were used.

\section{Insert Figure 1 around here}

To compute the spectral moments, the modulus of the short-term acoustic spectrum was computed with Digital Fourier Transform (512 points) for a time series of successive Hanning windows of $5 \mathrm{~ms}$, overlapping by $60 \%$, for the whole duration of the noise. Spectral moments were calculated with MATLAB ${ }^{\circledR}$, for the spectral envelope in the range going from $2000 \mathrm{~Hz}$ to $6500 \mathrm{~Hz}$, and with a linear decibel scale (dB). The low-frequency part was discarded to remove any potential influence of voicing. This computation consisted of the following steps (Forrest et al., 1988): (1) the spectrum modulus in $\mathrm{dB}$ in frequency range $(2000 \mathrm{~Hz}-6500 \mathrm{~Hz})$ was normalised in amplitude by the integral of this modulus over the frequency range; this normalization provides the spectrum with properties that are similar to those of a distribution of probability $\mathrm{P}(\mathrm{Sp})$ of a random variable $\mathrm{Sp}$; $(2)$ on this basis, the mean $\left(m_{S p}\right)$ of variable $S p$, its standard deviation $\left(\sigma_{S p}\right)$ and the $3^{\text {rd }}$ and $4^{\text {th }}$ moments $(\mathrm{M} 3(\mathrm{Sp})$ and $M 4(S p))$, from the centred variable $\left(S p-m_{S p}\right)$ were calculated; (3) finally the skewness and kurtosis parameters were calculated according to the classical formulas (skewness $=\mathrm{M} 3(\mathrm{Sp}) / \sigma_{\mathrm{Sp}}{ }^{3} ;$ kurtosis $\left.=\mathrm{M} 4(\mathrm{Sp}) / \sigma_{\mathrm{sp}}{ }^{4}\right)$. The spectral Centre Of Gravity (COG) was given by the mean value $m_{S p}$. The Centre Of Gravity gives information about the average of the frequency distribution of the spectral energy.

A high COG has been found for anterior phonemes. The skewness parameter informs about the right-left asymmetry of the spectral envelope: the higher the skewness the more the spectral energy is localized on low frequencies (left skewed) as in posterior phonemes. The interpretation of the kurtosis parameter is more 
complex (see DeCarlo, 1997). For Gaussian-like distributions of probability it informs about the shape of the curve $P(x)$ : the flatter the curve, the smaller the kurtosis. This corresponds to the way Forrest et al., 1988 interpreted this parameter. However, for more complex distributions, the kurtosis depends at the same time on the amplitude of the maximum and on the amplitude of the tails of the distribution. Hence, for a spectral envelope having several local maxima, kurtosis becomes a global way to characterize the shape of the noise envelope of the consonant.

On this basis, it is expected that, in case of reduction of the amplitude of tongue movements, the COG of the anterior consonants (i.e. the alveolars [t], [d], [s], [z], and the post-alveolars [5], [3]) should decrease, while their skewness should increase. For the back consonants $([\mathrm{k}, \mathrm{g}])$, the contrary is expected. Given the complexity of the relations between the distribution shape and the kurtosis, no specific predictions were made concerning the links between the pathology and the values of the kurtosis.

The analysis of the variation of the Klatt VOT, the COG, the skewness and the kurtosis across conditions (PREOP, POSTOP1, POSTOP2) was carried out qualitatively. These parameters were considered to provide a reliable assessment of the speech quality after surgery, when they accounted for the perceptual ratings of the stimuli and their evolutions after surgery.

\section{Articulatory parameters}

Ultrasound devices measure the distance between the tongue contour and the probe. Hence, ultrasound signals vary both with changes in the tongue position and with changes in the probe position. In order to make sure that the recorded signals 
informs strictly about changes in tongue position, it is required either that the probe never moves with respect to the head or that probe movements can be detected and corrected. A number of systems have been designed in the past years to maintain constant the position of the probe in relation to those of the head (Stone 2005; Scobbie et al., 2008). They all have been proved to be helpful and efficient. However, they are physically extremely constraining, and it is not possible to use them with patients who have undergone a surgery of the vocal tract and, often, a neck dissection.

A photography of the location of the probe was used to reposition the probe nearly at the same place across conditions (PREOP, POSTOP1, POSTOP2). In addition, during the recording a special attention was devoted to a careful visual control of the probe position. To check if the probe had kept constant during the production of each stimulus, a rectangle was drawn with a marker at the selected probe location on the patient's skin. However, since the probe was not physically constrained to stay at the same place, the possibility remains that the probe position has changed across stimuli and conditions. Part of the processing of the data was thus conceived in order to find a way to extract relevant information about changes in articulation across conditions, in spite of the lack of calibration. This has been largely inspired by the proposals made by Menard and colleagues in the Phonetic Laboratory of Montréal (Ménard et al., 2012).

Tongue contours were extracted and smoothed by a sixth degree polynomial function, using MATLAB ${ }^{\circledR}$. For the sequences in which the probe did not move much, it was possible to reliably evaluate the relative displacement and deformation of the tongue during the sequence. To do so a parameter that provides a global measure of the speed of deformation and elevation of the tongue contour in the plane defined by 
the ultrasound beam was developed. This parameter was measured as follows. For a given sequence the origin of the axis system in the plane was set at the location of the lowest point of all the contours taken together. In this plane a contour is defined a function $y=F(x)$, in which the $x$-axis is the horizontal axis, and $x$ varies in the interval $\left[\mathrm{x}_{\min }, \mathrm{x}_{\max }\right]$. The integral of function $\mathrm{F}$ for $\mathrm{x}$ varying within $\left[\mathrm{x}_{\min }, \mathrm{x}_{\max }\right]$ was computed and this integral was normalized by the difference $\left(\mathrm{x}_{\max }-\mathrm{x}_{\min }\right)$. The time derivative of this integral was calculated. This derivative was selected to be the parameter that measures the relative tongue shape changes during each sequence. We named this parameter "Speech of the Normalized Tongue Surface" (SNTS). The time variation of this parameter was approximated with a fourth degree polynomial function (see figure 2).

\section{Insert Figure 2 around here}

\section{Relevance of the acoustic and articulatory parameters}

The methodology to assess the relevance of the acoustic and articulatory parameters that are described above, was (1) to compute these parameters for each of the VCV sequences studied for each speaker, and (2) to investigate which of these parameters that show differences across recording conditions are compatible with the perceptual evaluations.

\section{Results}

For each consonant, the data recorded in the $/ \mathrm{i}-\mathrm{i} /$ and $/ \mathrm{a}-\mathrm{a} /$ contexts were pooled in a single data set, and no further analysis was made of the specificity of the vowel context. The $/ \mathrm{u}-\mathrm{u} / \mathrm{context}$ was not considered in this study because the 
protrusion of the lips tends to mask the spectral consequences of changes in tongue position.

\section{Perceptual analysis}

Perceptual ratings were first analysed for all consonants together and for each patient separately with a Friedman test, in order to evaluate the global perceptual effect of the "condition" factor (PREOP, POSTOP1, POSTOP2) in the /i-i/ and /a-a/ contexts taken together. There is a significant effect of this factor for both patients (P1: Friedman chi-squared $(2)=54.91, p<0.01 ; P 2$ : Friedman chi-squared $(2)=105.22$, $\mathrm{p}<0.01)$. Post hoc tests were then conducted to compare conditions pairwise. All differences were significant. The significance level was adjusted to 0.0167 for the post hoc tests, following the Bonferroni method. As expected, the perceptual ratings of both speakers' stimuli were the worst for the first post-surgery condition, i.e. a month after surgery (PREOP<POSTOP, PREOP<POSTOP2 and POSTOP1>POSTOP2). The worse perceptual scores were given for patient P2 as it can be seen in figure 4. Mean perceptual results are shown in Figures 3 and 4, respectively for patient $\mathrm{P} 1$ and $\mathrm{P} 2$.

\section{Insert Figures $3 \& 4$ around here}

\section{Acoustical analysis}

Results are presented in Figures 5 and 6, respectively for patient P1 and P2. For the alveolar and post-alveolar consonants, the decrease of the perceptual quality of the stimuli observed for both patients from condition PREOP to condition POSTOP1 is generally associated with a decrease of the COG and an increase of 
the skewness. This is in agreement with our expectations: after surgery the tongue is less mobile and the amplitude of the forward movements (associated with high spectral frequencies) is reduced. There are some exceptions: for patient P1 the perceptual quality of the post-alveolar [S] and [3] does improves from PREOP to POSTOP1 and the COG and the skewness are fairly constant; for patient P2, the perceptual quality of $[\mathrm{t}]$ and [d] decreases, while the COG increases and skewness decreases; for [3] produced by patient P2, the skewness does not increase from PREOP to POSTOP1, while COG and perceptual quality decrease. From condition POSTOP1 to condition POSTOP2, the perceptual quality tends to improve, without reaching the baseline (PREOP). This improvement is generally associated with an increase of the COG and a decrease of the skewness. Again there are some exceptions: for patient P1, the perceptual quality of consonants [C] and [3] remains stable and the COG and the skewness also; still for patient $\mathrm{P} 1$, the perceptual quality of $[\mathrm{s}]$ becomes worse in POSTOP2 than in POSTOP1, in spite of the fact that the COG increases and the skewness decreases.

For the velar consonants, the perceptual quality decreases for both patients from condition PREOP to condition POSTOP1 and improves from condition POSTOP1 to condition POSTOP2. The decrease of the perceptual quality is associated with a decrease of the COG and an increase of the skewness. This result suggests that in contrast to our expectations, the reduction of tongue mobility leads the patient to articulate velar consonants at a more posterior position, in the velopharyngeal region and not more forward in the palatal region. This can be explained by the fact that velopharyngeal sounds do not require as much tongue bunching than palato-velar ones. This strategy is consistent with observations made 
from patients after glossectomy in Savariaux et al. (2000). No clear relation could be found between the change of the kurtosis and the perceptual ratings.

In sum, in the majority of cases the COG and the skewness parameters provide information that is congruent with the perceptual rating: for all consonants, a decrease of the COG and an increase of the skewness reveal a decrease of the perceptual quality. However, three cases could not be described by these parameters for patient P2: the global evolution of the production of the stops [t] and [d] and the change from PREOP to POSTOP1 for the sibilant [3]. For patient P1, only one case could not be explained: the change of the production of the sibilant [s] from POSTOP1 to POSTOP2. Two other cases, []] and [3] of patient P1, did not show any decrease of the perception quality.

\section{Insert Figure $5 \& 6$ around here}

The next step was to seek for a measure that could account for these 4 cases not explained by spectral moments. The Klatt VOT was used and compared across conditions as illustrated in figures 7 . VOT variations across conditions are congruent with the perceptual ratings for $[t, d, 3]$ of patient $P 2$ : a lengthening of the Klatt VOT is associated with a decrease of quality (from PREOP to POSTOP1) and a reduction in the VOT values in POSTOP2 condition corresponds to the improvement of the quality from POSTOP1 to POSTOP2. For the sibilant [s] of patient P1 the Klatt VOT does not explain the decrease of the speech quality from POSTOP1 to POSTOP2. 


\section{Insert Figure 7 around here}

In sum, neither the spectral changes, which reflect changes in articulatory position, nor the Klatt VOT can explain the differences in perceptual ratings that suggest a decrease of speech quality from POSTOP1 to POSTOP2 in [s] for patient P1. In the next section, we will show that the time variation of the SNTS provides a possible account for this phenomenon.

\section{Articulatory analysis}

Figure 8 depicts the time variation of the SNTS parameter for the sequences /isi/ and /asa/ produced by speaker P1. The zero-crossings of these curves reflect changes in movement directions: an increase of the tongue surface indicates either an elevation of the tongue or more central position of the tongue; a decrease indicates either a flattening or a clear displacement towards the front of the back of the vocal tract. The extrema of these curves reflect the maximal speed in either direction. Hence, both number of zero-crossing and amplitude of the extrema are a way to assess the dynamics of tongue mobility, i.e. the capacity of the tongue to move in different directions within a same sequence.

From condition PREOP to POSTOP1 we observe clear differences in the SNTS time variation in /asa/. These changes in tongue dynamics contribute to the decrease of speech quality together with the changes in tongue position reflected by the modification of COG and skewness mentioned earlier and illustrated in Figure 5. This is not the case for /isi/. For both sequences, in condition POSTOP2, SNTS varies less and with smaller amplitude than in POSTOP1. This is consistent with the decrease of the speech quality observed from POSTOP1 to POSTOP2. 
Thus, the decrease of the speech quality observed for patient $\mathrm{P} 1$ from POSTOP1 to POSTOP2 condition in the sibilant [s] seems to be due to a reduction of the dynamics of tongue mobility which is captured by the SNTS time variation.

\section{Insert Figure 8 around here}

\section{Discussion}

On the basis of a perceptual evaluation carried out by five speech therapists, it is shown that the quality of the consonants produced in VCV sequences by the two patients who underwent hemiglossectomy was significantly lower one month after surgery (condition POSTOP1) than before surgery (condition PREOP), and it improved three months after surgery (condition POSTOP2) without reaching the quality of the pre-surgery condition. Three spectral moments, COG, skewness and kurtosis, were computed from the acoustic signal in the burst for the stops and in the frication noise for the sibilants. A specific parameter was extracted from the noncalibrated ultrasound data, the "Speed Normalised Tongue Surface" (SNTS) that should inform, in the absence of calibration of the ultrasound data, about the capacity of the subjects to move and shape the tongue within each VCV sequence.

Changes in the spectral moments COG and skewness corresponded with the perceptual evaluation of a large majority of the analyzed consonants in the $/ \mathrm{i}-\mathrm{i} /$ and /a-a/ contexts. The Kurtosis parameter did not provide reliable information that could be related to the perceptual ratings. Thus, the kurtosis parameter does not seem to 
be an efficient parameter to characterize pathological speech produced by glossectomized patients.

In the /a-a/ and /i-i/ contexts, the measures of the Klatt VOT were congruent with the perceptual evaluation when the latter could not be explained by spectral parameters i.e. [t, d, 3] for P2, with an exception though for the [s] of patient P1. It was found that changes in the SNTS parameter corresponded with changes in the perceptual evaluation of this consonant in the two vowel contexts.

The combined analysis of acoustic and articulatory parameters enable an interpretation of the causes of the decrease in speech quality and it change after surgery. For the majority of the productions, spectral changes measured by the COG and skewness were shown to be associated with the decrease of speech quality. These spectral changes can be interpreted as evidence for an inadequate tongue positioning: a lowering of the COG associated with an increase of the skewness postsurgery suggests that the location of the articulation of the consonant is more posterior. An increase of the Klatt VOT is associated with the fact that a longer duration is required to reach the physical conditions of a laminar airflow in the vocal tract after the release of the consonant. This can be due either to a decreased capacity for the patients to move the tongue rapidly or to problems with saliva related to a possible xerostomia. The articulatory evaluation shows that patient $\mathrm{P} 1$ has decreased capacity in post-operative conditions to shape the entire tongue correctly within the duration of a $\mathrm{V}-/ \mathrm{s} /-\mathrm{V}$ sequence. This articulatory parameter suggests for $\mathrm{P} 1$ a significant global stiffening of the tongue or a reduced strength in the tongue. These physical changes could not be noticed on the basis of the spectral parameters that we selected. 
Combining spectral and temporal acoustic analyses with ultrasound based articulatory measures enable an inference of the physical origins of the decrease of speech quality after surgery. This combination of parameters reveals impairments related either to the dynamics of tongue mobility or to the reduced capacities to position the tongue correctly in the anterio-posterior direction. This comprehensive functional assessment could help therapists to define the best possible rehabilitation protocol including specific tasks involving various kinds of tongue displacement and shaping.

\section{Conclusion}

The combination of acoustic, articulatory and perceptual data about the speech production of two patients who underwent hemiglossectomy has enabled a first evaluation of the relevance of some specific acoustic and articulatory parameters. The relevance has been evaluated by the capacity of these parameters to let emerge differences across recording conditions which follow perceptual judgements, and, second, by their capacity to suggest articulatory explanations for these differences. The COG and the spectral moments give information about tongue positioning that was relevant for speech quality. The Klatt VOT and articulatory measurement provide relevant information for the assessment of the sequences that could not be explained by spectral moments. The articulatory parameter named SNTS provides relevant information in terms of the dynamics of the mobility of the tongue. It has been extracted without any calibration of the ultrasound data. This is an important point, since calibration of the ultrasound data is often not possible with glossectomized patients in a clinical environment. 


\section{Acknowledgments}

The data were recorded at European Hospital Georges Pompidou in Paris in the ENT department (Head: Pr. Brasnu). The authors gratefully thank Lise Crevier-Buchman (European Hospital Georges Pompidou \& LPP, Paris), Benjamin Roustan, Thomas Hueber (Gipsa-lab) and Alan Wrench (Articulate Instruments) for their help at different stages of the work.

\section{Conflict of Interest Statement}

Audrey Acher, on behalf of Pascal Perrier, Christophe Savariaux and Cécile Fougeron declares that there is no conflict of interest. 


\section{References}

Articulate Instruments Ltd 2011. Articulate Assistant Advanced User Guide: Version 2.13. Edinburgh, UK: Articulate Instruments Ltd.

Articulate Instruments Ltd 2008. Ultrasound Stabilisation Headset Users Manual: Revision 1.4. Edinburgh, UK: Articulate Instruments Ltd.

Bressmann, T., Sader, R., Whitehill, T. L. \& Samman, N. (2004). Consonant intelligibility and tongue motility in patients with partial glossectomy. Journal of Oral and Maxillofacial Surgery, 62, 298-303.

Bressmann, T., Ackloo, E., Heng, C. L. \& Irish, J. C. (2007). Quantitative threedimensional ultrasound imaging of partially resected tongues. Otolaryngology-Head Neck Surgery, 136, 799-805.

Buchaillard, S., Brix, M., Perrier, P. \& Payan, Y. (2007). Simulations of the consequences of tongue surgery on tongue mobility: implications for speech production in post-surgery conditions. International Journal of Medical Robotics and Computer Assisted Surgery, 3, 252-261.

Bundgaard, T., Tandrup, O. \& Elbrønd, O. (1993). A functional evaluation of patients treated for oral cancer. A prospective study. International Journal of Oral and Maxillofacial Surgery, 22, 28-34.

DeCarlo, L. T. (1997). On the meaning and use of Kurtosis. Psychological Methods, 2, 292-307.

Dios, P. D., Feijoo, J. F., Ferreiro, M. C. \& Alvarez, J. A. (1994). Functional Consequences of Partial Glossectomy. Journal of Oral and Maxillofacial Surgery, 52, 12-14. 
Forrest, K., Weismer, G., Milenkovic, P. \& Dougall, R. N. (1988). Statistical analysis of word-initial voiceless obstruents: preliminary data. Journal of Acoustical Society of America, 84, 115-123.

Heller, K. S., Levy, J. \& Sciubba, J. J. (1991). Speech patterns following partial glossectomy for small tumors of the tongue. Head \& Neck, 13, 340-343.

Jesus, L. M. T. \& Shadle, C. H. (2002). A parametric study of the spectral characteristics of European Portuguese fricatives. Journal of Phonetics, 30, 437-464.

Jones, J. A. \& Munhall, K. G. (2003). Learning to produce speech with an altered vocal tract: the role of auditory feedback. Journal of the Acoustical Society of America, 113, 532-543.

Kazi, R., Prasad, V. M. N., Kanagalingam, J. Georgalas, C., Venkitaraman, R., Nutting, C. M., . . . Harrington, K. J. (2007). Analysis of formant frequencies in patients with oral or oropharyngeal cancers treated by glossectomy. International Journal of Language \& Communication Disorders, 42, 521-532.

Konstantinovic, V. S. \& Dimic, N. D. (1998). Articulatory function and tongue mobility after surgery followed by radiation therapy for tongue and floor of the mouth cancer patients. British Journal of Plastic Surgery, 51, 589-593.

Korpijaakko-Huuhka A. M., Söderholm A. L. \& Lehtihalmes, M. (1998). Long-lasting speech and oral-motor deficiencies following oral cancer surgery: a retrospective study. Logopedics Phoniatrics Vocology, 24, 97-106.

Laaksonen, J. P., Rieger, J., Harris, J. \& Seikaly, H. (2011). A longitudinal acoustic study of the effects of the radial forearm free flap reconstruction on sibilants produced by tongue cancer patients. Clinical Linguistics \& Phonetics, 25, 253-264.

Ligier, K., Belot, A., Launoy, G., Velten, M., Delafosse, P., Guizard, A. V, le réseau FRANCIM. (2011). Epidémiologie des cancers de la cavité buccale en France. Revue de Stomatologie et de Chirurgie Maxillo-Faciale, 112, 164-171. 
Lisker, L. \& Abramson, A. S. (1964). A cross-language study of voicing in initial stops: acoustical measurement. Word, 20, 384-422.

Meley, M. \& Barthelmé, E. (1987). Les cancers de la cavité buccale et de I'oropharynx, France:Masson.

Ménard, L., Aubin, J., Thibeault, M. \& Richard, G. (2012). Measuring Tongue Shapes and Positions with Ultrasound Imaging: A Validation Experiment Using an Articulatory Model. Folia Phoniatrica \& Logopaedica, 64, 64-72.

Savariaux, C., Perrier, P., Lebeau, J., Magaña, G. \& Dorange-Pattoret, C. (2000). Production de parole après traitements de cancers de la cavité endobuccale. Proceedings of the XXIIIrd Journées d'Etude de la Parole, 433-436, Aussois, France.

Schliephake, H. Schmelzeisen, R., Schönweiler, R., Schneller, T. \& Altenbernd, C. (1998). Speech, deglutition and life quality after intraoral tumor resection. A prospective study. International Journal of Oral and Maxillofacial Surgery, 27, 99-105.

Scobbie, J. M., Wrench, A. A. \& Van der Linden, M. (2008). Head-probe stabilisation in ultrasound tongue imaging using a headset to permit natural head movement. Proceedings of the Eighth International Seminar on Speech Production (ISSP), 373376, Strasbourg University, France.

Stevens, K. N. \& Klatt, D. H. (1974). Role of formant transitions in the voicedvoiceless distinction for stops. Journal of the Acoustical Society of America, 55, 653659 .

Stone, M. \& Davis, E. (1995). A head and transducer support system for making ultrasound images of tongue/jaw movement. Journal of the Acoustical Society of America, 980, 3107-3112.

Stone M. (2005). A Guide to Analyzing Tongue Motion from Ultrasound Images. Clinical Linguistics and Phonetics, 19, 455-502. 
Whalen, D. H., Iskarous, K., Tiede, M. K., Ostry, D. J., Lehnert-Lehouillier, H., Vatikiotis-Bateson E. \& Hailey D. S. (2005). The Haskins optically corrected ultrasound system (HOCUS). Journal of Speech Language and Hearing Research, 48, 543-553. 


\section{Figure Captions}

\section{Figure 1}

Representations of the 4 events labeled on the acoustic signal.

Top panel: [aka]; upper part: acoustic signal; lower part: wide band spectrogram.

Bottom panel: [asa]; upper part: acoustic signal; lower part: wide band spectrogram.

V_Off=Offset of the vowel preceding the consonant; N_On=Noise Onset; N_Off=Noise Offset; Laminar_On=Onset of the laminar flow after the consonant.

\section{Figure 2}

The Speed Normalized Tongue Surface (STNS) parameter.

Step 1: The upper tongue contour $y$ is approximated by a $6^{\text {th }}$ order polynomial function of the horizontal spatial dimension $\mathrm{x}$ (upper panel)

Step 2: The integral

of $y(x, t)$ from $x$ min to $x \max$ is computed and normalized by the distance (xmax-xmin); the resulting variable parameter is named Normalized Tongue Surface; an example of its variation in time is given in the left bottom panel

Step 3: The time derivative —of of is computed; it is named Speed Normalized Tongue Surface (STNS); an example of its variation in time is given in the right bottom panel.

\section{Figure 3}

Mean of the perceptual ratings for each utterance for the 3 conditions for patient P1. The 5 judges were asked to rank the quality of the stimuli from 1 (normal), 2 (slightly impaired), 3 (moderately impaired), 4 (strongly impaired) to 5 (unintelligible).

\section{Figure 4}

Mean of the perceptual ratings for each utterance for the 3 conditions for patient P2. The 5 judges were asked to rank the quality of the stimuli from 1 (normal), 2 (slightly impaired), 3 (moderately impaired), 4 (strongly impaired) to 5 (unintelligible). 


\section{Figure 5}

Mean of the acoustic measurements and perceptual ratings for each sequence for /a-a/ and /i-i/ contexts pooled for the 3 conditions for patient P1. Values along the $\mathrm{Y}$-axis are either without dimension (Perception score, Skewness, Kurtosis) or in $\mathrm{kHz}$ (COG).

\section{Figure 6}

Mean of the acoustic measurements and perceptual ratings for each sequence for /a-a/ and /i-i/ contexts pooled for the 3 conditions for patient P2. Values along the $\mathrm{Y}$-axis are either without dimension (Perception score, Skewness, Kurtosis) or in $\mathrm{kHz}$ (COG).

\section{Figure 7}

Evolution of Klatt VOT during time for consonants /s/ in /a-a/ and /i-i/ contexts pooled for patient $\mathrm{P} 1$ and /t, d, 3 / in /a-a/ and /i-i/ contexts pooled for patient P2.

\section{Figure 8}

Evolution of the "Speed Normalised Tongue Surface" for /asa/ (top) and /isi/ (bottom) pronounced by $\mathrm{P} 1$. 


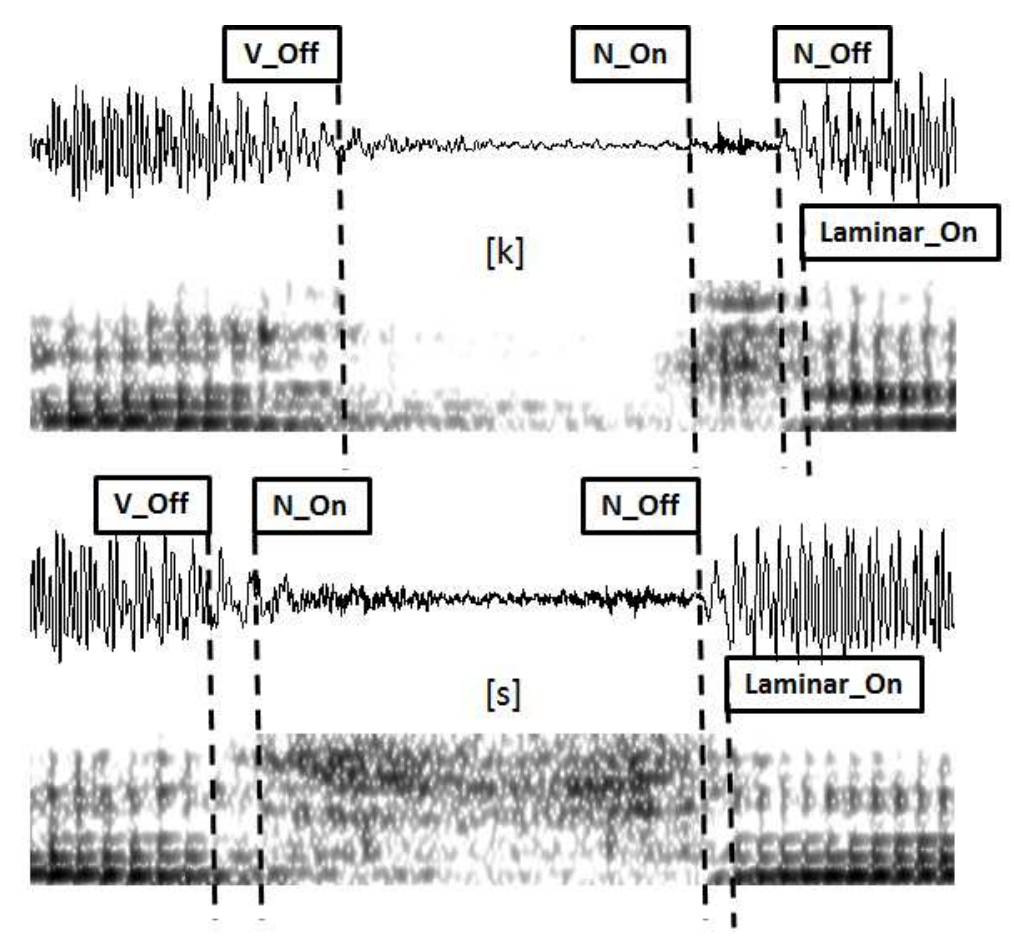

Figure 1

Representations of the 4 events labeled on the acoustic signal.

Top panel: [aka]; upper part: acoustic signal; lower part: wide band spectrogram.

Bottom panel: [asa]; upper part: acoustic signal; lower part: wide band spectrogram.

V_Off=Offset of the vowel preceding the consonant; N_On=Noise Onset; N_Off=Noise Offset; Laminar_On=Onset of the laminar flow after the consonant. 

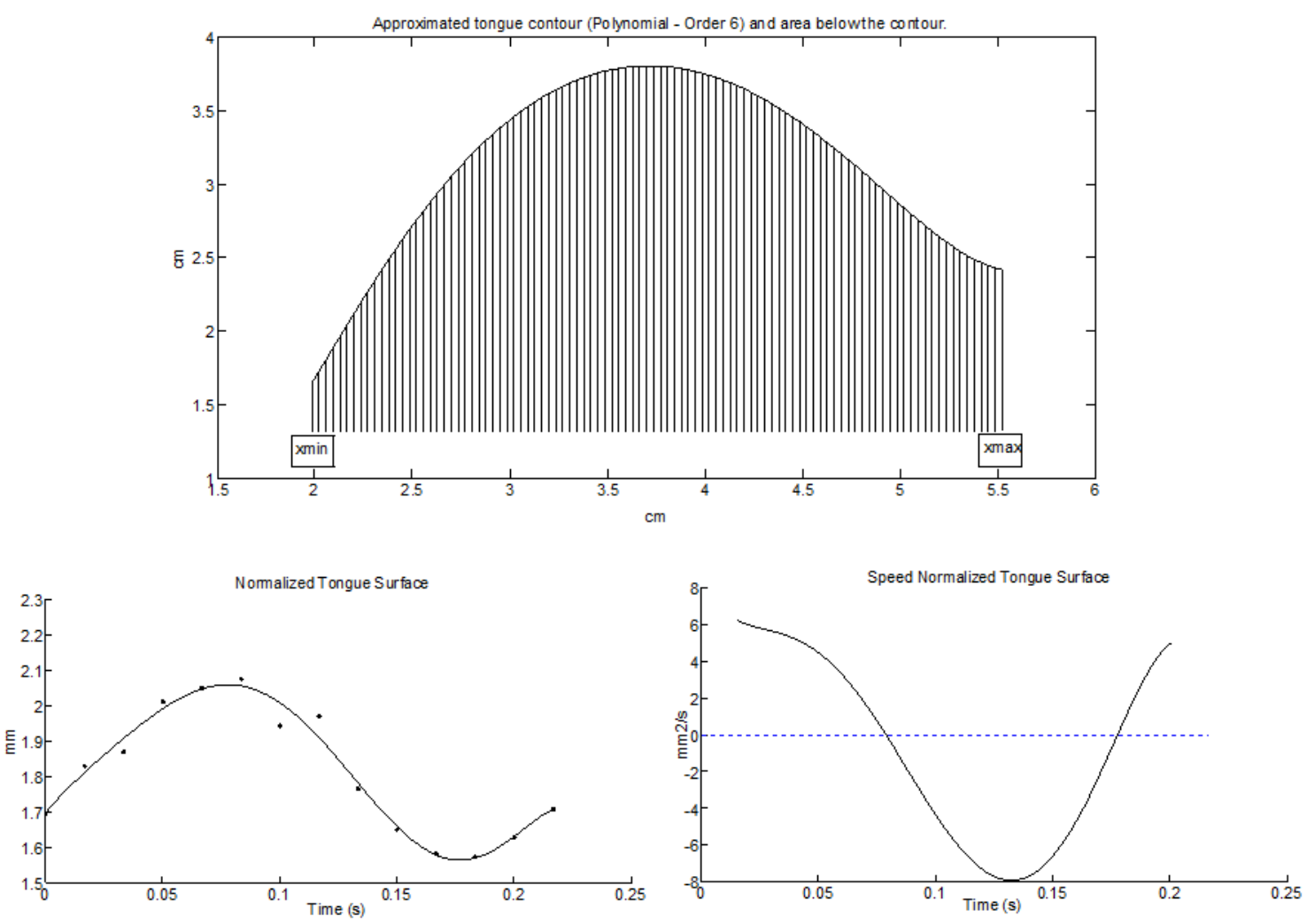

Figure 2

The Speed Normalized Tongue Surface (STNS) parameter.

Step 1: The upper tongue contour $y$ is approximated by a $6^{\text {th }}$ order polynomial function of the horizontal spatial dimension $x$ (upper panel)

Step 2: The integral

of $y(x, t)$ from $x$ min to $x$ max is computed and

normalized by the distance (xmax-xmin); the resulting variable parameter is named Normalized Tongue Surface; an example of its variation in time is given in the left bottom panel

Step 3: The time derivative is computed; it is named Speed Normalized Tongue Surface (STNS); an example of its variation in time is given in the right bottom panel. 


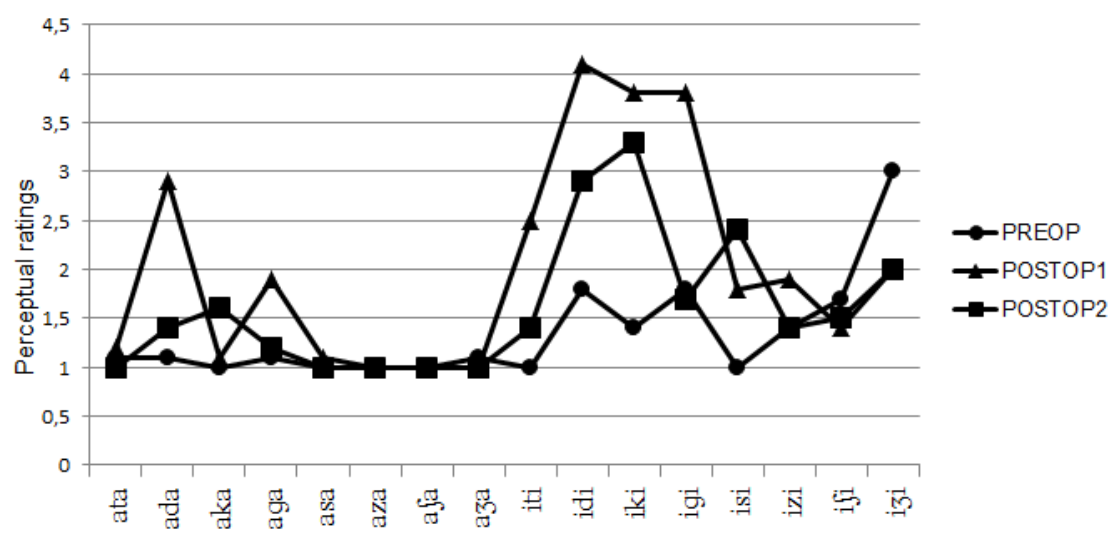

Figure 3

Mean of the perceptual ratings for each utterance for the 3 conditions for patient $\mathrm{P} 1$. The 5 judges were asked to rank the quality of the stimuli from 1 (normal), 2 (slightly impaired), 3 (moderately impaired), 4 (strongly impaired) to 5 (unintelligible).

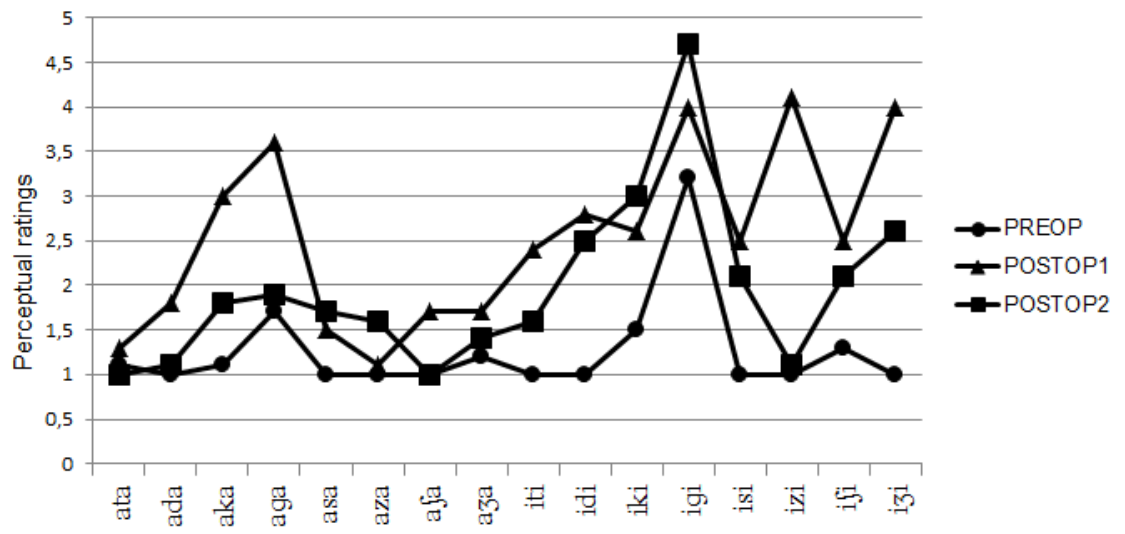

Figure 4

Mean of the perceptual ratings for each utterance for the 3 conditions for patient P2. The 5 judges were asked to rank the quality of the stimuli from 1 (normal), 2 (slightly impaired), 3 (moderately impaired), 4 (strongly impaired) to 5 (unintelligible). 


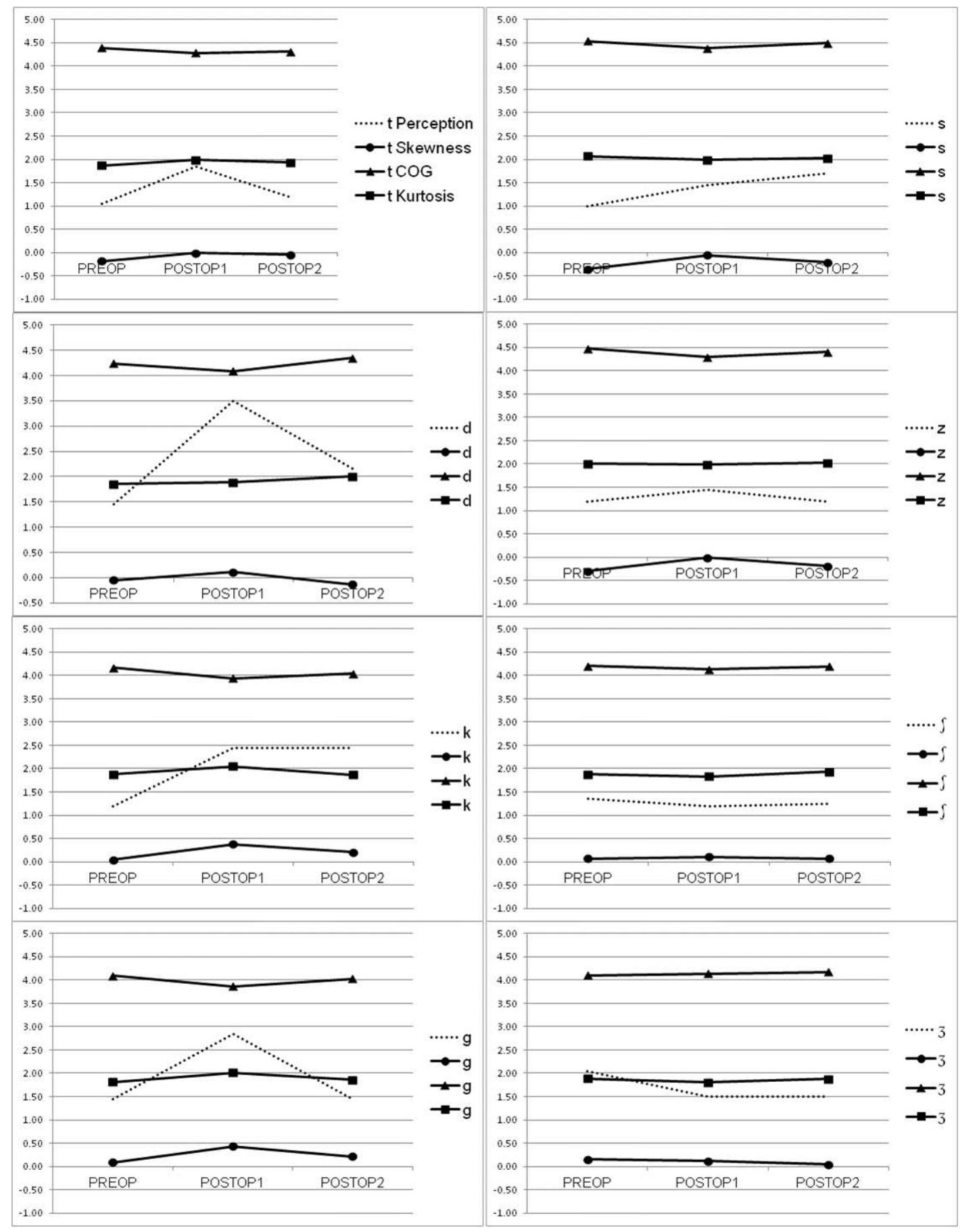

Figure 5

Mean of the acoustic measurements and perceptual ratings for each sequence for /a-a/ and /i-i/ contexts pooled for the 3 conditions for patient $\mathrm{P} 1$. Values along the $\mathrm{Y}$-axis are either without dimension (Perception score, Skewness, Kurtosis) or in $\mathrm{kHz}$ (COG). 


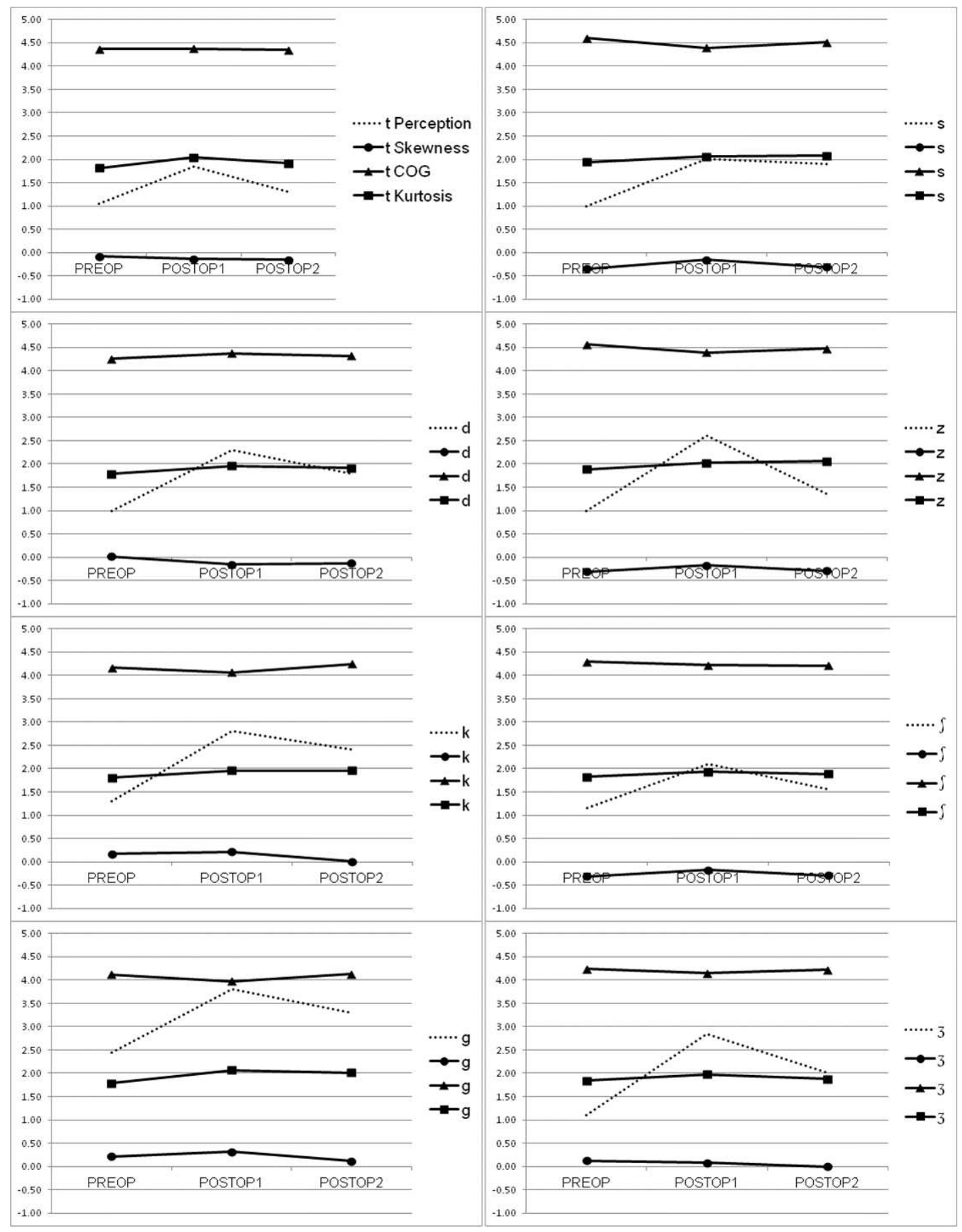

Figure 6

Mean of the acoustic measurements and perceptual ratings for each sequence for /a-a/ and /i-i/ contexts pooled for the 3 conditions for patient $\mathrm{P} 2$. Values along the $\mathrm{Y}$-axis are either without dimension (Perception score, Skewness, Kurtosis) or in $\mathrm{kHz}$ (COG). 


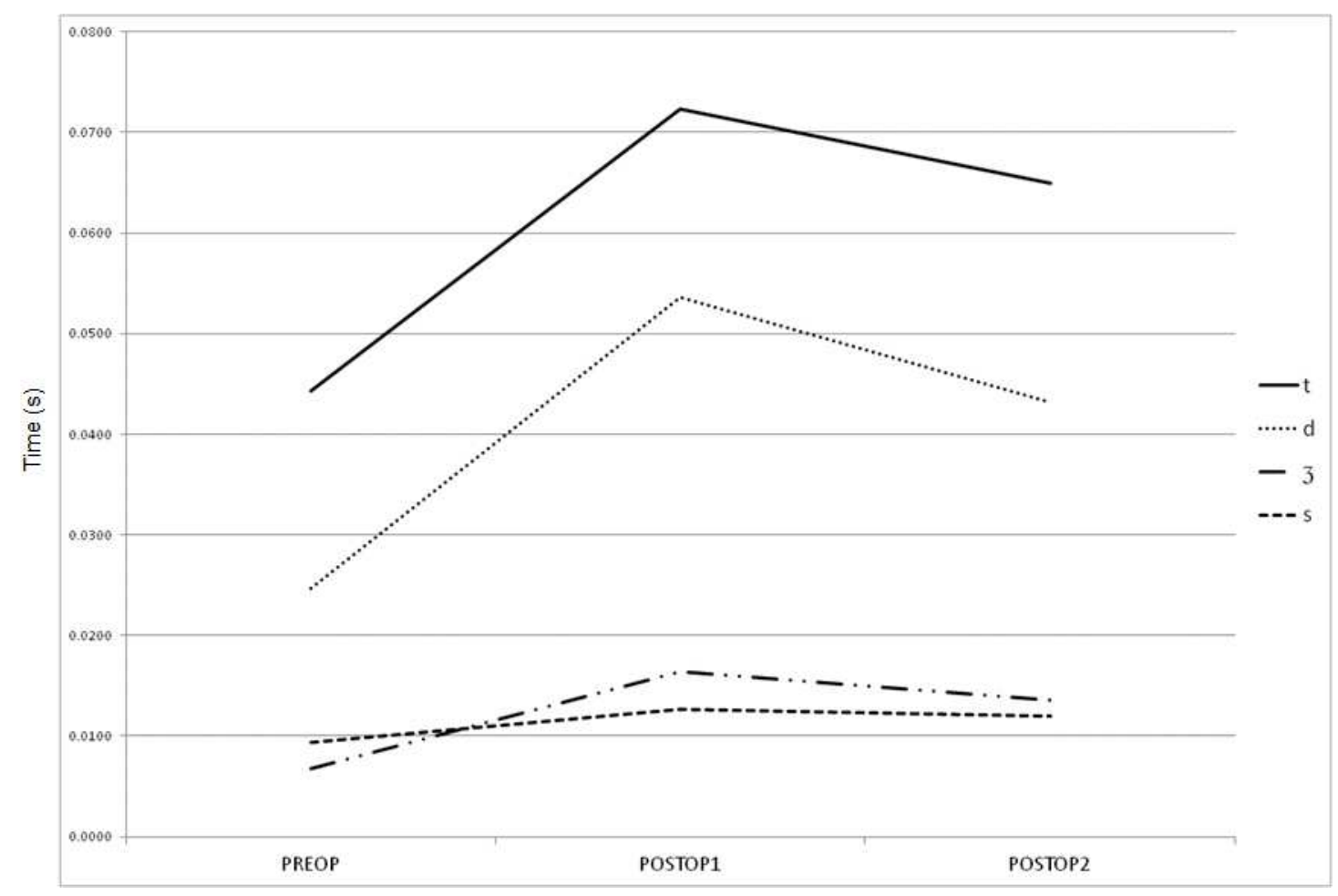

Figure 7

Evolution of Klatt VOT during time for consonants /s/ in /a-a/ and /i-i/ contexts pooled for patient $\mathrm{P} 1$ and /t, d, $3 /$ in /a-a/ and /i-i/ contexts pooled for patient P2. 

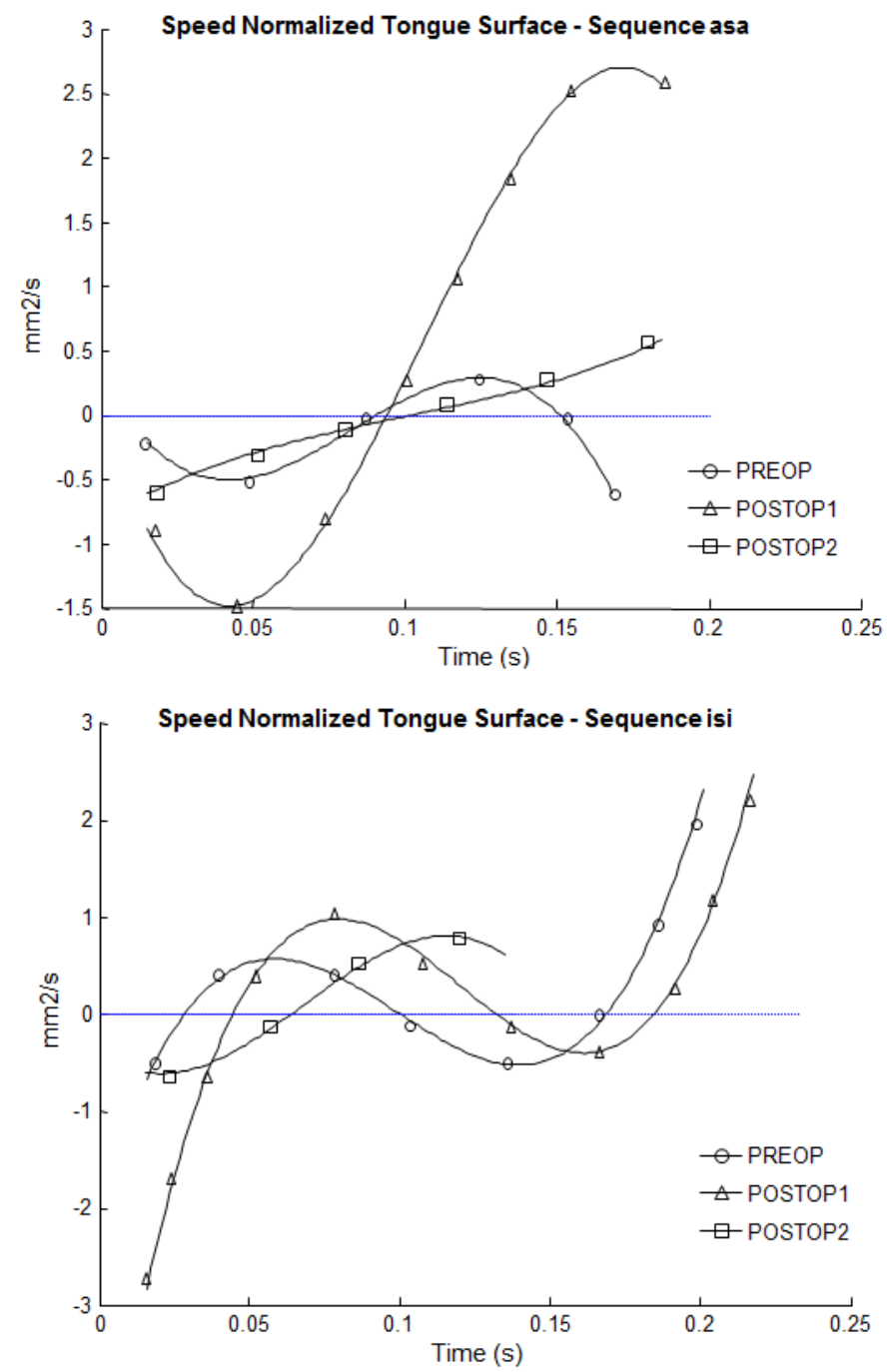

Figure 8

Evolution of the "Speed Normalised Tongue Surface" for /asa/ (top) and /isi/ (bottom) pronounced by P1. 